Original Article - Clinical Science

\title{
Glaucoma Australia educational impact study: a randomized short-term clinical trial evaluating the association between glaucoma education and patient knowledge, anxiety and treatment satisfaction
}

Simon E Skalicky FRANZCO PhD, ${ }^{1,2}$ Guy D'Mellow FRANZCO, ${ }^{3,4}$ Philip House FRANZCO, ${ }^{5}$ Eva Fenwick PhD ${ }^{6,7,8}$ and the Glaucoma Australia Educational Impact Study Contributors

1Discipline of Ophthalmology, University of Sydney, Sydney, Australia

${ }^{2}$ Departments of Ophthalmology and Surgery, Royal Melbourne Hospital, University of Melbourne, Australia

3Terrace Eye Centre, Brisbane, Queensland, Australia

4Greenslopes Hospital, Brisbane, Queensland, Australia

${ }^{5}$ Centre for Ophthalmology and Visual Science, The University of Western Australia, Perth, Australia

${ }^{6}$ Centre for Eye Research Australia, Royal Victorian Eye and Ear Hospital, University of Melbourne, Australia

${ }^{7}$ Singapore Eye Research Institute, Singapore National Eye Centre, Singapore ${ }^{8}$ Duke-NUS Medical School, Singapore, National University of Singapore, Singapore

Correspondence: Simon Skalicky, 32 Gisborne St East Melbourne Victoria, 3002, Australia

Email: seskalicky@gmail.com

This is the author manuscript accepted for publication and has undergone full peer review but has not been through the copyediting, typesetting, pagination and proofreading process, which may lead to differences between this version and the Version of Record. Please cite this article as doi: 10.1111/ceo.13016

This article is protected by copyright. All rights reserved. 
Short running title: Glaucoma Australia educational impact study

Received 28 March 2017; accepted 19 J une 2017

Conflict of interest: Drs Simon Skalicky, Philip House and Guy D'Mellow are honorary members of the Glaucoma Australia Ophthalmology Liaison Committee; Drs Simon Skalicky and Guy D'Mellow are Councillors for Glaucoma Australia.

Funding sources: A grant was received from the Eye Surgery Foundation, WA.

This paper was registered at the Australian and New Zealand Clinical Trial Registry ACTRN12616000180415

Keywords: Glaucoma, Education, Quality of life 


\section{ABSTRACT}

I mportance: Targeted education may impact glaucoma patients' clinical experience.

Background: To measure the impact of patient-centred glaucoma-related education on knowledge, anxiety and treatment satisfaction.

Design: Multicentre Australia-wide randomised clinical trial.

Participants: 101 newly-diagnosed glaucoma patients randomised 1:1 to intervention or control groups.

Methods: Those randomised to the Glaucoma Australia educational intervention received telephone-based counselling about glaucoma followed by mail out information, in addition to usual care and information from their treating ophthalmologist. The control group received only usual care and information from their treating ophthalmologist. Surveys were administered at baseline and 4-weeks following intervention. 
Main outcome measures: The Auckland Glaucoma Knowledge Questionnaire (AGKQ) measured glaucoma related knowledge. The Hospital Anxiety and Depression Scale (HADS) and Eye Drop Satisfaction Questionnaire (EDSQ) measured anxiety and patient treatment satisfaction, respectively.

Results: Mean age was $64.7 \pm 11.1$ years and $52(51.5 \%)$ were male. There was no noticeable increase in knowledge levels in the control group (mean difference: 0.04 logits, $P=0.7)$, compared with 0.49 logits $(P=0.02)$ increase in knowledge levels in the intervention group. Between group comparison showed a non-significant increase in knowledge ( 0.45 logits, $P=0.07$ ) comparing intervention participants with controls. Intervention participants experienced a statistically significant decrease in anxiety compared to controls $(-0.60$ logits, $P=0.02)$. No between-group difference was found in EDSQ scores.

Conclusions and Relevance: Patient-centred glaucoma-related education and support services may improve knowledge and can reduce anxiety for newly diagnosed glaucoma patients. All glaucoma patients should be adequately counseled about the nature of the disease and its management. 


\section{NTRODUCTION}

Glaucoma leads to significant functional disability including reduced driving ability, impaired quality of life (QoL), social isolation due to reduced social interaction, fear for the future and loss of independence. ${ }^{1-8}$ Glaucoma treatment often involves longterm topical medication, lifelong surveillance with serial optic nerve analysis and visual field testing, and sometimes multiple surgical procedures, all of which may negatively impact QoL. ${ }^{9,} 10$ In Australia 208,000 individuals had glaucoma in 2005; this is predicted to rise to 379,000 by $2025 .{ }^{11}$

Patient-focused educational interventions are increasingly used in the management of glaucoma. ${ }^{12}$ Educational interventions to improve general health literacy and knowledge about glaucoma have a small but significant impact on improving patient wellbeing, treatment satisfaction and levels of anxiety. ${ }^{13,14}$ Counselling explaining the nature of glaucoma and its prognosis, the need for ongoing monitoring and importance of the treatment paradigm may improve the patient's knowledge and experience. ${ }^{15}$ Patient education has been shown to improve adherence ${ }^{16,17}$ and better adherence has been linked to a better prognosis in glaucoma. ${ }^{18}$

Glaucoma Australia (GA) is the peak advocacy body in Australia for patients with glaucoma. In an effort to improve the overall patient understanding from early in the disease course, GA has devised a comprehensive patient-focused educational intervention. Administered by trained educators by telephone, this intervention is designed to improve patient comprehension, reduce anxiety and empower patients to make appropriate decisions regarding their glaucoma management. However, the impact of this intervention upon patients newly diagnosed with glaucoma has not yet been evaluated formally.

We conducted a randomised controlled trial (RCT) to evaluate the impact of the GA educational intervention for newly diagnosed glaucoma patients, in terms of glaucoma-related knowledge, anxiety and treatment satisfaction. 


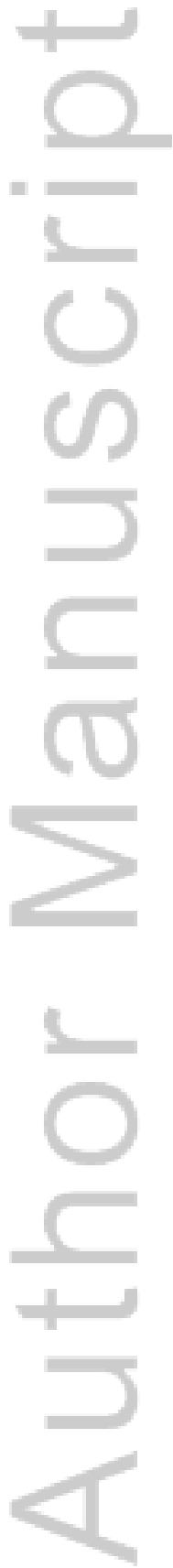

This article is protected by copyright. All rights reserved. 


\section{METHODS}

\section{Study Design and Registration}

This study was a prospective multicentred RCT and was registered at the Australian and New Zealand Clinical Trial Registry ACTRN12616000180415. The study protocol was approved by the Royal Australian and New Zealand College of Ophthalmology Human Research Ethics Committee (Study 48.14) and conformed to the tenets of the Declaration of Helsinki. All participants were fully informed of the reasons for the study, the study protocol and the process of anonymous storage and analysis of data. Participants were made fully aware that all participation was voluntary and would not influence the nature of the treatment or care they currently or subsequently would receive from their treating ophthalmologist. Informed consent was obtained from each participant.

\section{Study Participants}

Participants were recruited from subspecialty glaucoma clinics across Australia. All Australian ophthalmologists who manage patients with glaucoma were invited to register for participation in this study. Eligible subjects were approached consecutively by their ophthalmologist during regular clinic visits and invited to participate in the study.

\section{Inclusion Criteria}

Inclusion criteria were as follows: (1) age $\geq 30$ years; (2) a new diagnosis of open angle glaucoma in one or both eyes, made by their treating ophthalmologist (based on characteristic optic disc changes or glaucomatous visual field loss); (3) to be treated with intraocular pressure (IOP)-lowering glaucoma drops. Phakic and pseudophakic patients were included, as were patients with pigmentary glaucoma and pseudoexfoliation glaucoma. 


\section{Exclusion Criteria}

Exclusion criteria were as follows: (1) the inability to participate in telephone-based education sessions or questionnaire administration for any physical or psychological reasons, (2) the inability to speak and comprehend English fluently, (3) newly diagnosed glaucoma treated by other means (e.g. laser or surgery), (4) being treated with more than two ocular hypotensive medications, or (5) angle closure glaucoma, determined on gonioscopic evaluation by the treating ophthalmologist.

\section{Study Protocol}

\section{GA Questionnaire Administrator and Educator}

There was a single, dedicated GA educator and another single, dedicated GA questionnaire administrator involved in the implementation of this study. Both were appropriately trained prior to undertaking this RCT and subsequently assessed to ensure consistency in their approach to patients and educational intervention. The GA questionnaire administrator was masked as to the participant group.

\section{Randomisation}

Following consent and enrolment participants were randomised into two groups (Figure 1). Simple randomisation occurred using a randomly generated sequence allocating participants on a 1:1 ratio. This sequence and the randomisation process were concealed from the investigators, the GA questionnaire administrator and the GA educator in a password-encrypted dataset only accessible to a key GA staff member (GP).

\section{Baseline Survey}

Participants from both groups were contacted by the GA questionnaire administrator by telephone, who administered the baseline survey by telephone. The baseline 
survey was administered one to three weeks following initial glaucoma diagnosis by the treating ophthalmologist. It included demographic data and the following patient reported outcome (PRO) questionnaires:

1. The Auckland Glaucoma Knowledge Questionnaire (AGKQ) ${ }^{19}$ (22 items,

\section{Supplemental Material 1),}

2. The anxiety component of the Hospital Anxiety and Depression Scale $(\mathrm{HADS})^{20}$ (7 items, Supplemental Material 2).

\section{Intervention Group}

The intervention group received usual care for their treating ophthalmologist at the time of diagnosis, and subsequently received the GA educational intervention via telephone and mail.

\section{Control Group}

The control group received only usual care and information from their treating ophthalmologist; the nature of education provided by the treating ophthalmologist was not captured and is likely to have varied among practitioners. After the final survey was conducted they received the same GA educational intervention as the intervention group.

\section{Intervention}

\section{A. Education Session One}

Education session one occurred 1 day following the baseline survey. This was undertaken by telephone contact to the participant by the GA educator. The duration ranged from five to 20 minutes, with 10 minutes being an average duration. The following prescribed topics were discussed, depending on the participant level of interest and time available:

1. The nature of glaucoma 
2. Risk factors for glaucoma

3. The relationship between IOP and glaucoma

4. Management strategies in glaucoma

5. The importance of adherence to medication

6. The need for lifelong treatment and monitoring

7. Eye-drop instillation techniques and dosing times

8. Family members who should be screened

9. Other resources for patients and their relatives/carers (e.g. relevant websites) The information for the above topics was taken from the following reference material (all written and/or approved by members of the Glaucoma Australia Expert Medical Panel):

1. Glaucoma - the Thief of Sight pamphlet

2. What You Should Know About Eye Drops pamphlet

3. Glaucoma Australia Fact Sheets

4. Glaucoma Australia website pages

5. Glaucoma Runs in Families brochure and resource cards

B. Mail-out

1-2 days following education session one the following information was received by mail (see Supplementary Material 3):

1. Glaucoma - the Thief of Sight pamphlet

2. What You Should Know About Eye Drops pamphlet

3. A recent Glaucoma Australia newsletter (standardised for all participants)

C. Education Session Two

Education session two occurred approximately two weeks following receipt of mailout information, and was administered by the GA educator. The duration ranged from three to 10 minutes, with six minutes being an average duration. The same references as used for education session one were used. Its purpose was to check if the participant had received and read the mail out, to allow them to ask further 
questions, to seek clarification or to raise any other concerns about glaucoma, their treatment or any other related matter.

\section{Final Survey}

The final survey was administered by the GA questionnaire administrator four weeks after the baseline survey to both groups (following completion of the GA education in the intervention group). It included:

1. The AGKQ readministered

2. The HADS-A readministered

3. The Eye Drop Satisfaction Questionnaire (EDSQ) ${ }^{21}$ (20 items)

\section{Survey Contents}

The AGKQ comprises 22 true/false statements regarding patient-relevant glaucoma facts. ${ }^{19}$ Devised by a working group and subsequently internally and externally validated, ${ }^{19}$ it is an appropriate metric for evaluating Australian glaucoma patients' knowledge of glaucoma. Higher scores represent higher levels of glaucoma-specific knowledge.

The HADS is a 14-item PRO designed to measure the traits for anxiety (HADS-A; 7 items) and depression (HADS-D; 7 items). Each item is answered on a Likert scale scoring 0-3. The scale has been widely used to measure anxiety and depression in a variety of clinical illness scenarios (e.g. cancer, ${ }^{22}$ musculoskeletal ${ }^{23}$ and neurological ${ }^{24,} 25$ illness); including glaucoma. ${ }^{14}$ It has been strongly validated and Rasch analysed. ${ }^{25,}{ }^{26}$ In this study only the HADS-A was used, as the effect of the education on anxiety level was our main focus. A HADS-A raw score $\geq 9$ indicates abnormal anxiety levels, 7-8 borderline, and $\leq 6$ normal. ${ }^{25}$

The EDSQ is a self-administered 20-item PRO designed to assess patients' satisfaction and adherence to eye-drop treatment, covering six domains: patient concerns about treatment, concerns about disease, satisfaction with patient-clinician 
relationship, positive beliefs, treatment convenience and self-declared adherence. ${ }^{21}$, ${ }^{27}$ It was used primarily as an instrument to measure patient concerns with drops, difficulty and negative symptoms associated with drops and satisfaction with their treatment. A higher overall score signifies a higher level of satisfaction.

These PROs were chosen because they have been previously validated, are directly applicable to the Australian health-care context and are targeted to specific dimensions of interest (i.e. disease knowledge, disease-related anxiety and treatment satisfaction).

\section{Outcome Measures}

As this study primarily evaluates the impact of a specific format of glaucoma information, the primary outcome measure is change in glaucoma knowledge (measured by the AGKQ) over the study period. The secondary outcome measures are change in glaucoma-related anxiety measured by the HADS-A and final treatment satisfaction measured by the EDSQ.

\section{Rasch Analysis}

Rasch analysis ${ }^{28}$ was used to assess the psychometric properties of the AGKQ, HADS-A and EDSQ using the Andrich rating scale model ${ }^{29}$ with Winsteps software (version 3.92.1), Chicago, Illinois, USA. ${ }^{30}$ Rasch analysis is a form of item response theory where ordinal ratings of questionnaires are transformed to estimates of interval measures (expressed in log of the odds units, or logits). The numbers reported for person measures range from a negative number to a positive number. Rasch analysis also provides substantial insight into the psychometric properties of a scale, including how well the response categories function, scale precision, unidimensionality, item 'fit', targeting of item 'difficulty' to person 'ability', and differential item functioning (DIF; item bias). ${ }^{31}$ 
Scale precision indicates the capacity of the instrument to discriminate between differing levels of the underlying construct (e.g. knowledge). Precision is usually determined using sample-based person separation index (PSI) for which a value of $>2.0$ is desired. This represents the capacity of a scale to distinguish at least three levels of the construct. ${ }^{32}$

\section{Statistical Analysis}

All analyses were performed using Stata 13.1 (Statacorp LP, College Station, TX, USA). Patient characteristics at baseline between the control and intervention groups were compared. Since no significant differences in patient characteristics were found between the two groups (Table 2), paired t-tests were utilized to compare the within-group difference in knowledge and anxiety in the control and intervention participants.

To calculate the between group difference in knowledge and anxiety, independent sample t-tests were used to compare the delta change in pre-post knowledge and anxiety levels between the control and intervention participants. Eye drop satisfaction at follow-up was also compared using independent sample t-test.

\section{Sample Size and Power Calculations}

Power calculations were performed as follows. A significance level (a) of $5 \%$ and a power $(1-\beta)$ of $80 \%$ were chosen for two-tailed, non-paired student $t$ test to compare final results in the AGKQ scores between two groups. Predicted mean scores and standard deviation for the two groups were based on data from DaneshMayer HV et al. ${ }^{21}$ In that study the data were found to be normally distributed however median scores and interquartile ranges were supplied. Newly diagnosed glaucoma patients without education had a median score of 16/22 (interquartile range 13-18), while controls (without glaucoma) had a median score of 13 (interquartile range 10-14). There was a marginal increase in knowledge for 
established glaucoma patients without any educational intervention (17/22). Based on the median difference of 3 points in that study, one can perhaps assume the effect of the educational intervention to raise the score by $2-3$ points (ie to 18.5/22). Assuming a $10 \%$ drop out rate we calculated that a sample size of $90-100$ individuals were required. Of note this is the same number of individuals recruited to most clinical studies evaluating anxiety related to clinical disease using the HADS.

\section{RESULTS}

Study enrolment occurred from February 2015 to October 2016. 168 patients with glaucoma from 13 centres across six States and Territories in Australia were consecutively assessed for eligibility in this study. Of these 66 were excluded from the study and 102 were enrolled (Figure 2). 54 were randomized to the intervention arm and 48 to the control arm; one from the control arm was lost to follow up. The distribution of patients per treating ophthalmologist and per state is outlined in Table $\mathbf{1 .}$

Table 2 Outlines the results of Rasch analysis of the three PROs. One item in the AGKQ was removed as it displayed DIF for time, and five items were removed from the EDSQ to resolve DIF, misfit and multidimensionality. Following this, the psychometric properties of the two scales were adequate although precision was suboptimal. The HADS-A did not require any amendments, although again precision was suboptimal.

The mean age (SD) of the 101 participants was 64.7 (11.1) years and 52 (51.5\%) were male. The mean baseline knowledge and anxiety levels were 1.70 (SD 1.41; range -5.10 to 4.98 ) logits and -2.81 (SD 2.09; range -5.87 to 5.31) logits, respectively. No significant differences in patient characteristics were found between the control and intervention groups (Table 3). 


\section{Effect of the intervention on outcomes}

Table 4 shows the within-group difference in knowledge and anxiety for the control and intervention participants. There was no noticeable increase in knowledge level in participants in the control group at study completion (mean difference: 0.04 logits, $\mathrm{P}$ $=0.7)$, compared to a significant 0.49 logits $(P=0.02)$ increase in knowledge level in participants who underwent the intervention. This corresponds approximately to a 3 point increase in AGKQ score, consistent with our a priori assumptions for power calculation (Supplemental Material 4). In addition, a non-significant increase in anxiety level was noted in controls ( 0.27 logits; $P=0.08$ ), while intervention participants experienced a non-significant decrease $(-0.33$ logits; $P=0.1)$ in anxiety level.

Between group comparison (Table 4) of the mean delta change in knowledge and anxiety levels between control and intervention participants showed that intervention participants did not result in a significant increase in level of knowledge ( 0.45 logits, $P=0.07$ ) compared with controls. However they experienced a statistically significant decrease in anxiety level at study completion compared to control participants (-0.60 logits, $P=0.02$ ). This corresponds approximately to a 2.25 drop in raw scores in the HADS-A (Supplemental Material 4).

No difference was found in EDSQ scores at study completion between the two groups (mean score 0.95 and 0.85 for the control and intervention group, respectively, $p=0.8$; range -1.00 to 4.98 logits).

\section{DISCUSSION}

In our multicentre RCT evaluating the effectiveness of a glaucoma education intervention, we found significant within-group improvement in glaucoma-specific knowledge in the intervention but not the control group. However, a between-group comparison found no difference in improvement of glaucoma knowledge, despite the 
intervention group demonstrating a non-significant greater improvement in knowledge than controls ( $0.49 \vee 0.04$ logits, $p=0.07$ ), correlating with approximately a three-point increase in the knowledge questionnaire (score out of 22). A difference of three points is substantial, corresponding with the difference in knowledge between established glaucoma patients and healthy controls in the Danesh-Meyer et al study, a cross-sectional clinical study from New Zealand that compared the degree of glaucoma knowledge among 208 established glaucoma patients, 100 new glaucoma suspects and 100 healthy controls using the AGKQ. ${ }^{19}$ In our study a significant improvement in anxiety level was detected in the intervention group compared to controls. Our findings suggest the importance of disease-specific education strategies to improve patient knowledge, awareness of disease and anxiety levels.

Our findings are in keeping with the EQUALITY pre- and post-intervention crosssectional study, which evaluated the impact of a telemedicine informational program on knowledge and awareness of glaucoma. ${ }^{13}$ The EQUALITY study demonstrated improvement in knowledge for all items of their purpose-designed knowledge questionnaire; however unlike our study, the EQUALITY study did not have a randomised control arm for comparison, and did not use a pre-validated PRO. At four weeks a significant improvement in anxiety level was detected in the intervention group compared to control group, which exhibited a worsening of anxiety level. This may be because improvements in disease specific knowledge empower patients to make appropriate decisions about their care and thus reduce anxiety about the unknown. Correction of misconceptions regarding prognosis and influence of treatment may allay patients' fears of future blindness and social withdrawal. ${ }^{14}$ This is in keeping with current literature that demonstrates telephonebased counselling can improve anxiety scores in a variety of chronic health conditions. ${ }^{33-36}$ The increase in anxiety level among controls may reflect further time 
to contemplate the new diagnosis, its implications for future vision, medical treatment and lifestyle implications.

No significant difference was detected between groups for treatment satisfaction, both in total EDSQ logit score and item-by-item analysis (data not shown), indicating that the structured information and patient contact facilitated by GA has no influence on treatment satisfaction. The items within the EDSQ refer mostly to the patient's satisfaction with topical therapy (e.g. inconvenience, side effects) or their perception of their relationship with their physician; neither of these may be substantially altered by communication from a third party. It is also possible that the lack of detected difference may be because measurement precision of the EDSQ was suboptimal (PSI 1.6), or because there were various physicians treating the participants in this study which may have introduced noise into the measurement. Increasingly it is recognised that patients who acquire information regarding glaucoma from sources external to their physician have greater adherence to and persistence with therapy. ${ }^{37}$ Educational interventions that improve health literacy have a positive influence on subjects' adherence to therapy, potentially more so than other factors such as ethnicity, age, gender, and economic status. ${ }^{38,39}$ It was beyond the scope of this study, primarily administered by telephone-trained trained supportive staff, to directly measure treatment adherence and persistence. Some items of the EDSQ indirectly reflect adherence (items 15-17), however items 16 and 17 were removed from analysis due to DIF and misfit respectively. We were unable to assess the direct impact of the GA educational intervention on patient adherence. Strengths of this study include the randomised, controlled, masked prospective design, the use of validated PROs and involvement of multiple sites across Australia. It is a unique RCT to directly evaluate the impact of patient education in glaucoma; to evaluate the impact of supportive, third party advocacy services in glaucoma; and to evaluate how counselling interventions can influence disease related knowledge or anxiety in glaucoma. Such services are increasingly important in the care of 
glaucoma patients, and are generally provided free of charge to patients, offering support and promotion of disease awareness.

However, the results of this study must be interpreted within the context of some limitations. For example, it was difficult to predict the required sample size precisely a priori, as the chosen primary outcome measure (AGKQ) has not been used extensively in prior studies. However the basis for our a priori assumptions was subsequently supported by the findings of our study (Supplemental Material 4). All PROs were chosen based on robust prior metrics of validity and reliability, however Rasch analysis within this cohort revealed that not all items within the PROs conformed to the desired criteria; one item was removed from the AGKQ and five from the EDSQ. The PROs had suboptimal PSI, indicating they lacked ideal discrimination among individuals of different ability levels. The data from PROs was subjective, and can be influenced by mood, recall bias and other non-clinical influences; these factors have been minimised by randomisation and Rasch analysis. ${ }^{7} 40$ As the majority (85-87\%) of this cohort was of Caucasian ethnicity, these results cannot be extrapolated to other ethnic groups.

There was a non-significant difference in baseline anxiety levels between control and intervention participants. This difference is difficult to explain and may reflect stochastic variation; patients were randomised, and masked to randomisation at the time of baseline survey. This difference in scores reduced on follow-up, with nonsignificant rise in anxiety levels in controls, and non-significant decline in anxiety in intervention participants. Importantly, however, the difference between groups of change in anxiety level was significant ( 0.60 logits, $p=0.02$ ). It is possible that the increase in anxiety level in the control group is reflecting measurement bias, and may simply be due to being in the control arm of this study. However, to minimise 
this patients were reassured they would receive the information on study completion.

In this study an attention placebo, where GA staff would spend an equal amount of telephone contact-time with control participants but provide general counselling instead of glaucoma education, was not implemented. The addition of such a placebo might be beneficial in reducing any potential Hawthorne effect, determining whether the improvements noted were due to the specific intervention, or just more time interacting with the patient. Further studies evaluating the differential influence of varying the content of the education, and the time and nature of the interaction, would be of merit. In addition, four weeks may be too brief a time frame to fully appreciate the effect of education for a life-long disease; further studies evaluating the longer-term influence of education and support from an advocacy organisation like GA would be of merit.

In conclusion, a patient-centred, glaucoma-related education intervention provided by advocacy and support services may improve knowledge and reduce anxiety for newly diagnosed patients with glaucoma in the short term. Given the relatively low cost and resource requirements of such an intervention, it is likely to be an important additional component of routine clinical practice for patients newly diagnosed with glaucoma. Further studies evaluating its influence on treatment adherence and persistence, and the cost-effectiveness of the service would be of value.

\section{Acknowledgements}

We would like to extend our sincere thanks to Geoff Pollard, National Executive Officer of Glaucoma Australia and the Glaucoma Australia staff for their help administering the study. 


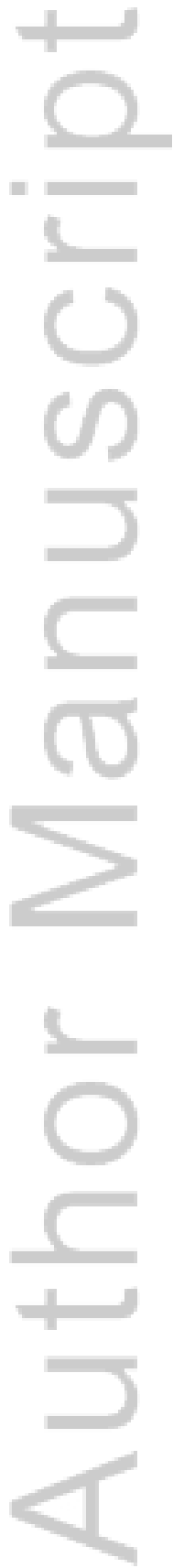

This article is protected by copyright. All rights reserved. 


\section{REFERENCES}

1. Burton R, Crabb DP, Smith ND, Glen FC and Garway-Heath DF. Glaucoma and reading: exploring the effects of contrast lowering of text. Optom Vis Sci. 2012; 89: 1282-7.

2. Crabb DP, Smith ND, Rauscher FG, et al. Exploring eye movements in patients with glaucoma when viewing a driving scene. PloS one. 2010; 5: e9710.

3. - Glen FC, Crabb DP, Smith ND, Burton R and Garway-Heath DF. Do patients with glaucoma have difficulty recognizing faces? Investigative ophthalmology \& visual science. 2012; 53: 3629-37.

4. Lahav K, Levkovitch-Verbin H, Belkin M, Glovinsky $Y$ and Polat U. Reduced mesopic and photopic foveal contrast sensitivity in glaucoma. Arch Ophthalmol. 2011; 129: 16-22.

5. Ramulu PY, Swenor BK, J efferys J L, Friedman DS and Rubin GS. Difficulty with out-loud and silent reading in glaucoma. Investigative ophthalmology \& visual science. 2013; 54: 666-72.

6. Ramulu PY, West SK, Munoz B, Jampel HD and Friedman DS. Glaucoma and reading speed: the Salisbury Eye Evaluation project. Arch Ophthalmol. 2009; 127: 82-7.

7. Skalicky S and Goldberg I. Depression and quality of life in patients with glaucoma: a cross-sectional analysis using the Geriatric Depression Scale-15, assessment of function related to vision, and the Glaucoma Quality of Life-15. Journal of glaucoma. 2008; 17: 546-51.

8. Janz NK, Wren PA, Guire KE, et al. Fear of blindness in the Collaborative I nitial Glaucoma Treatment Study: patterns and correlates over time. Ophthalmology. 2007; 114: 2213-20.

9. Quaranta L, Riva I, Gerardi C, Oddone F, Floriano I and Konstas AG. Quality of Life in Glaucoma: A Review of the Literature. Adv Ther. 2016; 33: 959-81. 
10. Janz NK, Wren PA, Lichter PR, et al. The Collaborative I nitial Glaucoma Treatment Study: interim quality of life findings after initial medical or surgical treatment of glaucoma. Ophthalmology. 2001; 108: 1954-65.

11. Dirani M, Crowston JG, Taylor PS, et al. Economic impact of primary openangle glaucoma in Australia. Clinical \& experimental ophthalmology. 2011; 39: 62332.

12. Cate H, Bhattacharya D, Clark A, Fordham R, Notley C and Broadway DC. Protocol for a randomised controlled trial to estimate the effects and costs of a patient centred educational intervention in glaucoma management. BMC ophthalmology. 2012; 12: 57.

13. Rhodes LA, Huisingh CE, McGwin G, J r., et al. Eye Care Quality and Accessibility Improvement in the Community (EQUALITY): impact of an eye health education program on patient knowledge about glaucoma and attitudes about eye care. Patient Relat Outcome Meas. 2016; 7: 37-48.

14. Kong XM, Zhu WQ, Hong J X and Sun XH. Is glaucoma comprehension associated with psychological disturbance and vision-related quality of life for patients with glaucoma? A cross-sectional study. BMJ open. 2014; 4: e004632.

15. Do AT, Pillai MR, Balakrishnan V, et al. Effectiveness of Glaucoma Counseling on Rates of Follow-up and Glaucoma Knowledge in a South Indian Population. Am J Ophthalmol. 2016; 163: 180-9 e4.

16. Sleath B, Blalock SJ, Carpenter DM, et al. Ophthalmologist-patient communication, self-efficacy, and glaucoma medication adherence. Ophthalmology. 2015; 122: 748-54.

17. Newman-Casey PA, Dayno M and Robin AL. Systematic Review of Educational Interventions to I mprove Glaucoma Medication Adherence: an update in 2015. Expert Rev Ophthalmol. 2016; 11: 5-20. 
18. Sleath B, Blalock S, Covert $D$, et al. The relationship between glaucoma medication adherence, eye drop technique, and visual field defect severity. Ophthalmology. 2011; 118: 2398-402.

19. Danesh-Meyer HV, Deva NC, Slight C, et al. What do people with glaucoma know about their condition? A comparative cross-sectional incidence and prevalence survey. Clinical \& experimental ophthalmology. 2008; 36: 13-8.

20. Zigmond AS and Snaith RP. The hospital anxiety and depression scale. Acta psychiatrica Scandinavica. 1983; 67: 361-70.

21. Regnault A, Viala-Danten M, Gilet $H$ and Berdeaux G. Scoring and psychometric properties of the Eye-Drop Satisfaction Questionnaire (EDSQ), an instrument to assess satisfaction and compliance with glaucoma treatment. BMC ophthalmology. 2010; 10: 1.

22. Lambert SD, Pallant JF, Boyes AW, King MT, Britton B and Girgis A. A Rasch analysis of the Hospital Anxiety and Depression Scale (HADS) among cancer survivors. Psychological assessment. 2013; 25: 379-90.

23. Harter M, Reuter K, Gross-Hardt K and Bengel J. Screening for anxiety, depressive and somatoform disorders in rehabilitation--validity of HADS and GHQ-12 in patients with musculoskeletal disease. Disability and rehabilitation. 2001; 23: 73744.

24. Forjaz MJ, Rodriguez-Blazquez C, Martinez-Martin P and Longitudinal Parkinson's Disease Patient Study G. Rasch analysis of the hospital anxiety and depression scale in Parkinson's disease. Movement disorders : official journal of the Movement Disorder Society. 2009; 24: 526-32.

25. Gibbons CJ, Mills RJ, Thornton EW, et al. Rasch analysis of the hospital anxiety and depression scale (HADS) for use in motor neurone disease. Health and quality of life outcomes. 2011; 9: 82.

26. Cameron IM, Scott NW, Adler M and Reid IC. A comparison of three methods of assessing differential item functioning (DIF) in the Hospital Anxiety Depression 
Scale: ordinal logistic regression, Rasch analysis and the Mantel chi-square procedure. Quality of life research : an international journal of quality of life aspects of treatment, care and rehabilitation. 2014.

27. Nordmann JP, Baudouin C, Renard JP, Denis P, Regnault A and Berdeaux G. Identification of noncompliant glaucoma patients using Bayesian networks and the Eye-Drop Satisfaction Questionnaire. Clinical ophthalmology. 2010; 4: 1489-96.

28. Rasch G. Probabilistic models for some intelligence and attainment tests. Chicago: University of Chicago, 1960.

29. Andrich D. Rating formulation for ordered response categories. Psychometrica. 1978; 43: 561-73.

30. Linacre J M. A user's guide to Winsteps/Ministeps Rasch-Model Computer Programs. Chicago, IL.: MESA press, 2014.

31. Lamoureux E and Pesudovs K. Vision-specific quality-of-life research: a need to improve the quality. Am J Ophthalmol. 2011; 151: 195-7 e2.

32. Bond TG and Fox CM. Applying the Rasch model: fundamental measurement in the human sciences. London: Lawrence Erlbaum Associates, 2001.

33. Gambatesa M, D'Ambrosio A, D'Antini D, et al. Counseling, quality of life, and acute postoperative pain in elderly patients with hip fracture. J Multidiscip Healthc. 2013; 6: 335-46.

34. Lovejoy TI. Telephone-delivered motivational interviewing targeting sexual risk behavior reduces depression, anxiety, and stress in HIV-positive older adults. Ann Behav Med. 2012; 44: 416-21.

35. Chan $\mathrm{CH}, \mathrm{Ng} \mathrm{EH}, \mathrm{Chan} \mathrm{CL}, \mathrm{Ho}$ and Chan TH. Effectiveness of psychosocial group intervention for reducing anxiety in women undergoing in vitro fertilization: a randomized controlled study. Fertil Steril. 2006; 85: 339-46.

36. Traeger AC, Hubscher M, Henschke N, Moseley GL, Lee H and McAuley JH. Effect of Primary Care-Based Education on Reassurance in Patients With Acute Low 
Back Pain: Systematic Review and Meta-analysis. JAMA Intern Med. 2015; 175: 73343.

37. Friedman DS, Hahn SR, Gelb L, et al. Doctor-patient communication, healthrelated beliefs, and adherence in glaucoma results from the Glaucoma Adherence and Persistency Study. Ophthalmology. 2008; 115: 1320-7, 7 e1-3.

38. Juzych MS, Randhawa S, Shukairy A, Kaushal P, Gupta A and Shalauta N. Functional health literacy in patients with glaucoma in urban settings. Arch Ophthalmol. 2008; 126: 718-24.

39. Muir KW, Santiago-Turla C, Stinnett SS, et al. Health literacy and adherence to glaucoma therapy. Am J Ophthalmol. 2006; 142: 223-6.

40. Warrian KJ, Spaeth GL, Lankaranian D, Lopes J F, Steinmann WC. The effect of personality on measures of quality of life related to vision in glaucoma patients. $\mathrm{Br}$ J Ophthalmol. 2009; 93: 310-5. 


\section{FIGURES}

Figure 1: Study outline

Figure 2: Participant recruitment and enrolment

\section{SUPPLEMENTARY MATERI AL}

Supplemental material 1: The Auckland Glaucoma Knowledge Questionnaire

Supplemental Material 2: The Hospital Anxiety and Depression Scale - anxiety component

Supplemental Material 3: A. Glaucoma - the Thief of Sight pamphlet; B. What You Should Know About Eye Drops pamphlet; C. A recent Glaucoma Australia newsletter (standardised for all participants)

Supplemental material 4: A. Ogival relationship between raw AGKQ scores (y-axis) and Rasch person measures in logits (x-axis). Higher raw and Rasch AGKQ scores indicate greater glaucoma-related knowledge. This figure shows that a 0.45 logit increase on the AGKQ questionnaire in intervention compared to control participants is approximately equivalent to a 3 point increase (or getting 3 additional questions correct) in raw AGKQ scores. B. Ogival relationship between raw HADS-A scores (y-axis) and Rasch person measures in logits (x-axis). Lower raw and Rasch HADS-A scores indicate less anxiety symptoms. This figure shows that a 0.65 logit decrease on the HADS-A in intervention compared to control participants is approximately equivalent to a 2.25 decrease in raw HADS-A scores. 


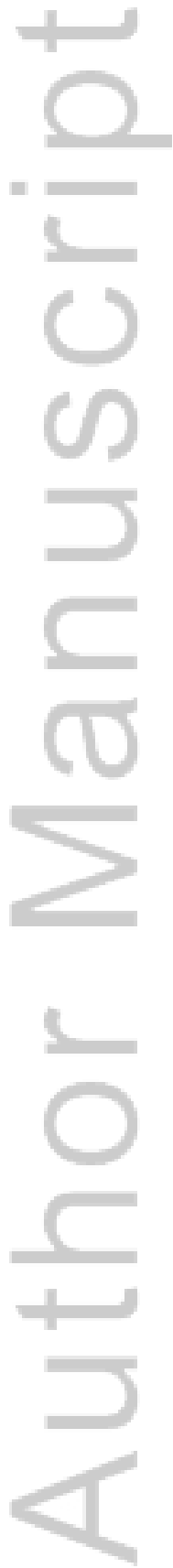

This article is protected by copyright. All rights reserved. 


\section{TABLES}

Table 1: Number of patients recruited per treating ophthalmologist and per state

\begin{tabular}{ccccc}
\hline Ophthalmologist & $\begin{array}{c}\text { No. of } \\
\text { Participants } \\
\text { recruited }\end{array}$ & $\begin{array}{c}\text { No. in } \\
\text { intervention } \\
\text { group }\end{array}$ & State & $\begin{array}{c}\text { State } \\
\text { total }\end{array}$ \\
\hline 1 & 4 & 1 & NSW & 27 \\
2 & 7 & 2 & NSW & \\
3 & 1 & 1 & NSW & \\
4 & 9 & 4 & NSW & \\
5 & 2 & 1 & NSW & \\
6 & 4 & 1 & NSW & 5 \\
\hline 7 & 5 & 3 & Qld & 5 \\
\hline 8 & 12 & 8 & SA & 12 \\
\hline 9 & 4 & 3 & Vic & 39 \\
10 & 11 & 4 & Vic & \\
11 & 3 & 2 & Vic & \\
12 & 21 & 11 & Vic & \\
\hline 13 & 20 & 13 & WA & 21 \\
14 & 1 & 1 & WA & \\
\hline
\end{tabular}


Table 2: Fit parameters of the EDSQ, AGKQ and the HADS-A compared to the Rasch model, before and after modification ( $n=101$ )

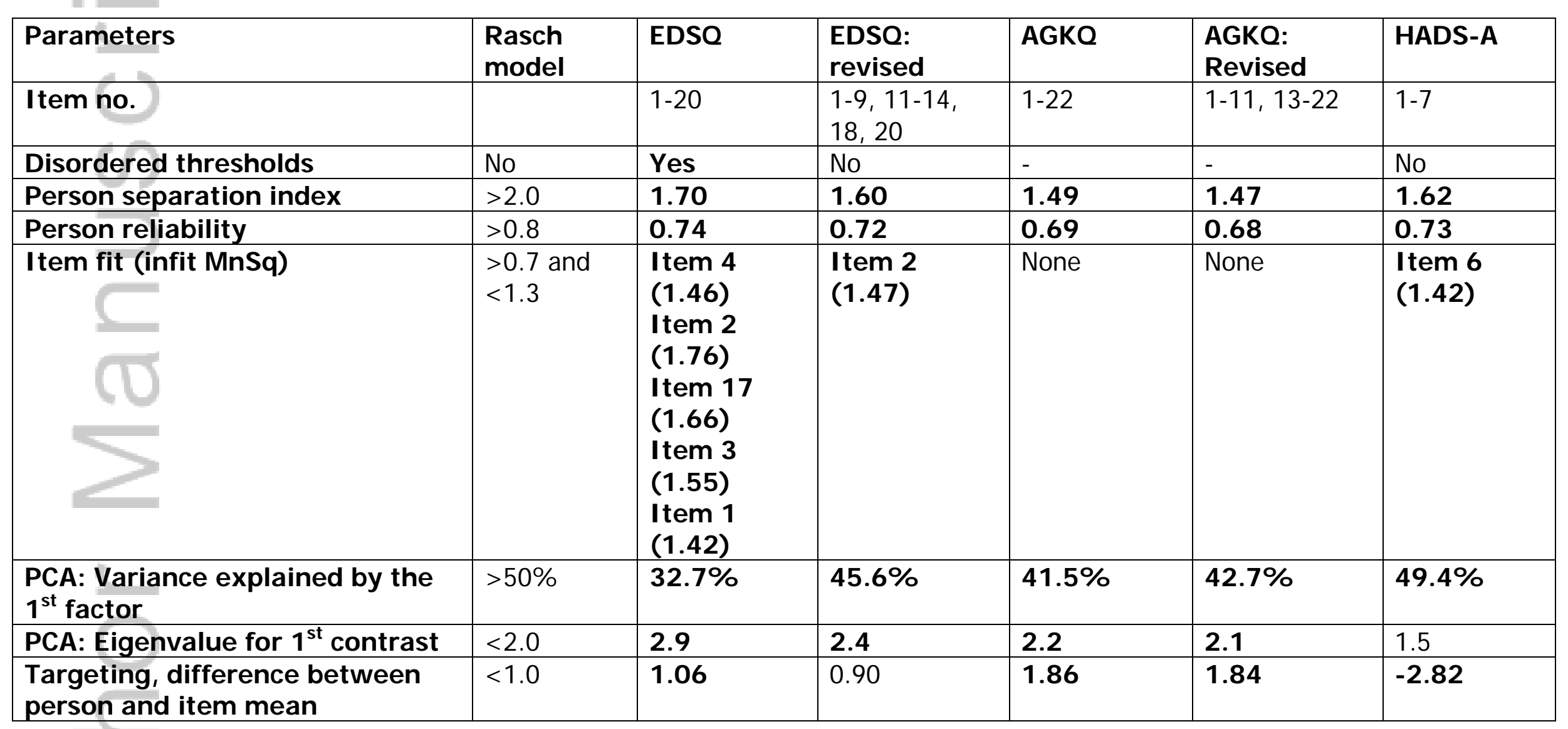




\begin{tabular}{|c|c|c|c|c|c|c|}
\hline DI F* & $\begin{array}{l}<1.0 \\
p>0.05\end{array}$ & $\begin{array}{l}\text { Age (Item } \\
\text { 12) }\end{array}$ & None & $\begin{array}{l}\text { Time (Item } \\
\text { 12) }\end{array}$ & None & None \\
\hline \multicolumn{7}{|c|}{$\begin{array}{l}\text { AGKQ=Auckland Glaucoma Knowledge Questionnaire; DI F=Differential Item Functioning; EDSQ=Eye Drop Satisfaction } \\
\text { Questionnaire; HADS-A=Hospital Anxiety and Depression Scale - Anxiety subscale; MnSq=Mean Square; PCA=Principal } \\
\text { Components Analysis } \\
\text { Note: Bolded values indicate misfit to the Rasch model; Shaded columns indicate scales that achieved acceptable fit to the Rasch } \\
\text { model. } \\
\text { *DIF was tested for age group (<65 years, } \geq 65 \text { years), gender, time (baseline, follow-up), and intervention vs. control group. }\end{array}$} \\
\hline
\end{tabular}

This article is protected by copyright. All rights reserved. 
Table 3: Comparison of baseline patient characteristics between controls and intervention

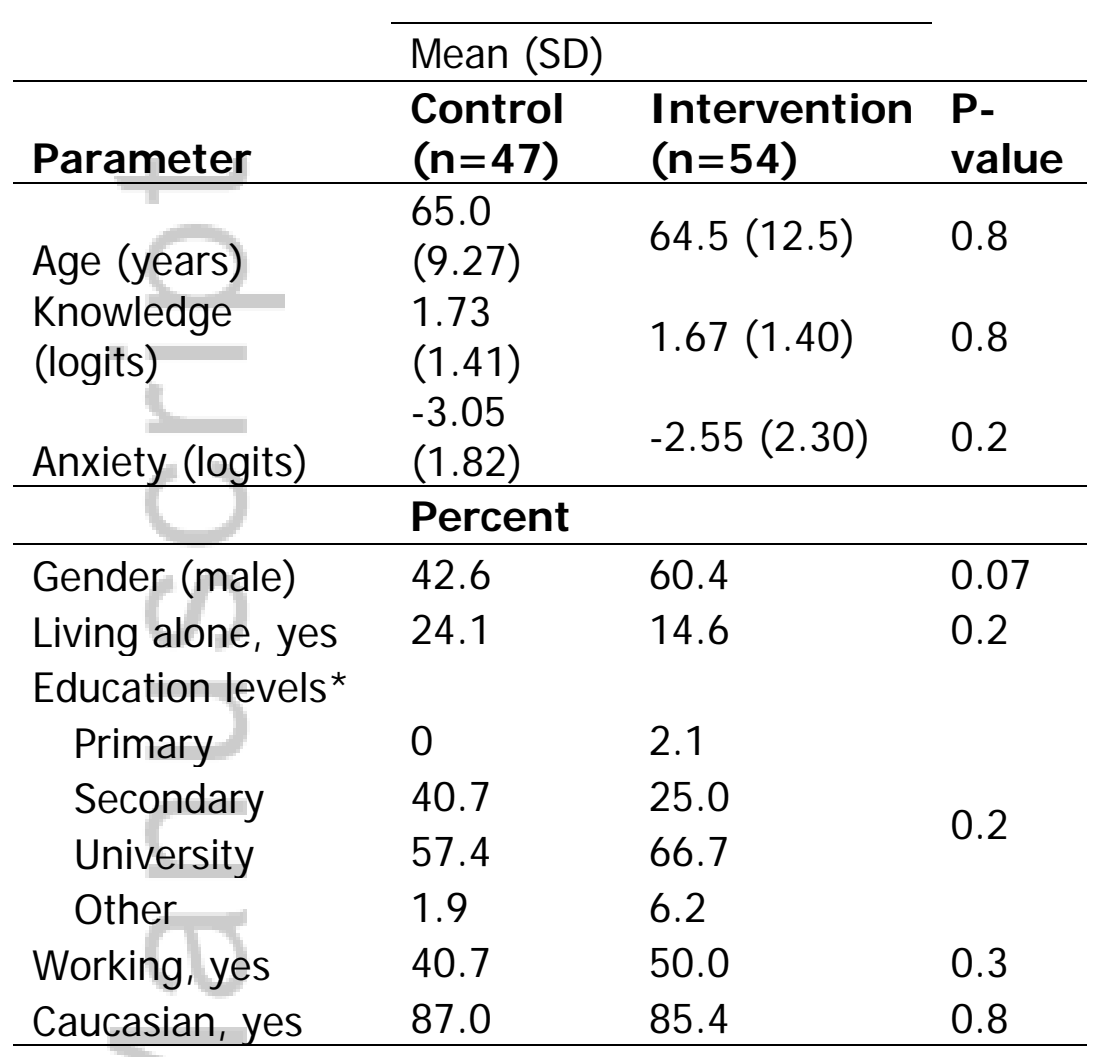

*Fisher's exact test for small counts 
Table 4: Knowledge and anxiety level outcomes: within group and between group comparison

Within group comparison: baseline $v$ follow-up scores

\begin{tabular}{|c|c|c|c|c|}
\hline $\begin{array}{l}\text { Outcome } \\
\text { Control partic }\end{array}$ & N & Baseline score & $\begin{array}{l}\text { Follow-up } \\
\text { score }\end{array}$ & P-value \\
\hline Knowledge & 47 & 1.73 & 1.77 & 0.7 \\
\hline Anxiety & 47 & -3.05 & -2.78 & 0.08 \\
\hline \multicolumn{5}{|l|}{ Intervention } \\
\hline Knowledge & 54 & 1.67 & 2.16 & 0.02 \\
\hline Anxiety & 54 & -2.55 & -2.88 & 0.1 \\
\hline \multicolumn{5}{|c|}{$\begin{array}{l}\text { Between group comparison: mean change in control } v \\
\text { intervention participants }\end{array}$} \\
\hline 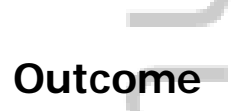 & $\mathbf{N}$ & $\begin{array}{l}\text { Mean change } \\
\text { (Control) }\end{array}$ & $\begin{array}{l}\text { Mean change } \\
\text { (intervention) }\end{array}$ & P-value \\
\hline <nowledge & 101 & 0.04 & 0.49 & 0.07 \\
\hline Anxiety & 101 & 0.27 & -0.33 & 0.02 \\
\hline
\end{tabular}


Day 0

Randomisation

Day 1

Intervention group

Control group

Day 4-5

Day $6 \quad \begin{gathered}\text { Education session } 1 \\ \text { (telephone education) }\end{gathered}$

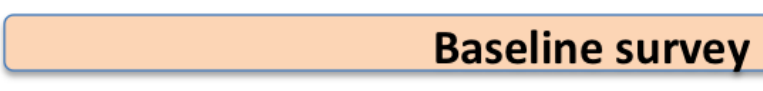

Day 7

Mail out
(information pack)

Day 10

Education session 2 (more questions/concerns)

Day 28

Final survey

Study closes

Day 30

Education session 1 (telephone education)

Day 32

Day 35

\begin{tabular}{|c|}
\hline $\begin{array}{c}\text { Mail out } \\
\text { (information pack) }\end{array}$ \\
\hline $\begin{array}{c}\text { Education session } 2 \\
\text { (more questions/concerns) }\end{array}$ \\
\hline
\end{tabular}

Fig 1 
13 Ophthalmologists participated

(6 states/territories across Australia)

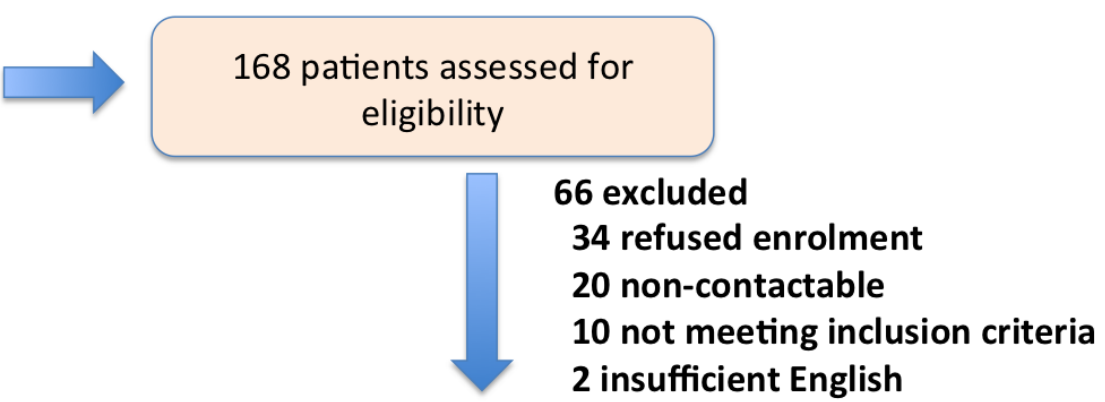

102 Randomized

\section{1:1 Randomization}

Intervention arm $n=54$

Control arm $n=48$

1 lost to follow up

47 included in analysis

Fig 2

This article is protected by copyright. All rights reserved. 


\section{University Library}

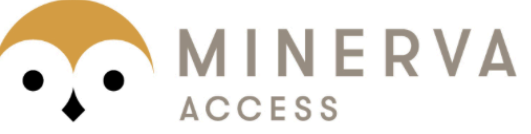

A gateway to Melbourne's research publications

Minerva Access is the Institutional Repository of The University of Melbourne

Author/s:

Skalicky, SE;D'Mellow, G;House, P;Fenwick, E

Title:

Glaucoma Australia educational impact study: a randomized short-term clinical trial evaluating the association between glaucoma education and patient knowledge, anxiety and treatment satisfaction

Date:

2018-04-01

Citation:

Skalicky, S. E., D'Mellow, G., House, P. \& Fenwick, E. (2018). Glaucoma Australia educational impact study: a randomized short-term clinical trial evaluating the association between glaucoma education and patient knowledge, anxiety and treatment satisfaction. CLINICAL AND EXPERIMENTAL OPHTHALMOLOGY, 46 (3), pp.222-231. https:// doi.org/10.1111/ceo.13016.

Persistent Link:

http://hdl.handle.net/11343/293307 